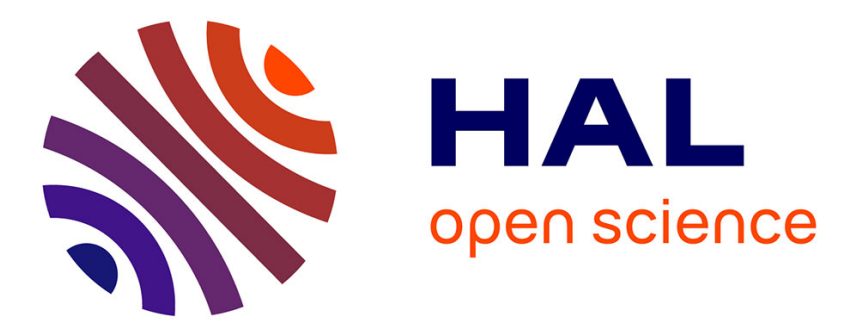

\title{
2-Dimensional Wavelet Packet Spectrum for Texture Analysis
}

Abdourrahmane Atto, Yannick Berthoumieu, Philippe Bolon

\section{To cite this version:}

Abdourrahmane Atto, Yannick Berthoumieu, Philippe Bolon. 2-Dimensional Wavelet Packet Spectrum for Texture Analysis. IEEE Transactions on Image Processing, 2013, pp.1-9. 10.1109/TIP.2013.2246524 . hal-00766713

\section{HAL Id: hal-00766713 https://hal.science/hal-00766713}

Submitted on 18 Dec 2012

HAL is a multi-disciplinary open access archive for the deposit and dissemination of scientific research documents, whether they are published or not. The documents may come from teaching and research institutions in France or abroad, or from public or private research centers.
L'archive ouverte pluridisciplinaire HAL, est destinée au dépôt et à la diffusion de documents scientifiques de niveau recherche, publiés ou non, émanant des établissements d'enseignement et de recherche français ou étrangers, des laboratoires publics ou privés. 


\title{
2-Dimensional Wavelet Packet Spectrum for Texture Analysis
}

\author{
Abdourrahmane M. ATTO ${ }^{1}$, Yannick BERTHOUMIEU ${ }^{2}$, Philippe BOLON ${ }^{3}$,
}

\begin{abstract}
The paper derives a 2-Dimensional spectrum estimator from some recent results on the statistical properties of wavelet packet coefficients of random processes. It provides an analysis of the bias of this estimator with respect to the wavelet order. The paper also discusses the performance of this wavelet based estimator, in comparison with the conventional 2-D Fourier-based spectrum estimator on texture analysis and content based image retrieval. It highlights the effectiveness of the wavelet based spectrum estimation.
\end{abstract}

Keywords - 2-D Wavelet packet transforms; Random fields; Spectral analysis, Spectrum estimation, Similarity measurements.

\section{INTRODUCTION}

W AVELET transforms have become appealing alternatives to the Fourier transform for image analysis and processing. Indeed, the availability of wavelet atoms with different shapes and different characteristics (vanishing moments, compact support, symmetric or non-symmetric, etc), as well as the wavelet multi-scale decomposition scheme make wavelets enough flexible for concise/sparse description of a wide class of images. In this respect, wavelet transforms are, at present time, extensively used for the description and the retrieval of image features, in many imaging systems such as those involved in machine vision, medical imaging, biometrics, radar and geoscience applications, etc.

In contrast with this extensive use of wavelets as a representation tool, spectral analysis by using wavelet transforms has not received much interest in the literature whereas spectral analysis plays a crucial role for characterizing and understanding textures and natural images.

Spectrum analysis is strongly connected to the distribution of variances in the frequency domain. In this respect, references [1], [2], [3], [4] have considered the wavelet framework in order to derive wavelet based spectra from subband variances computed at different scales. This yields a wavelet spectrum definition that can differ from the conventional definition of the Fourier based spectral density function. Indeed, most wavelet functions have their Fourier frequency supports spreading over large size supports so

\footnotetext{
1 Université de Savoie - Polytech Annecy-chambéry, LISTIC, Abdourrahmane.Atto@univ-savoie.fr

2 Université de Bordeaux, IPB, IMS, CNRS UMR 5218, Yannick.Berthoumieu@ims-bordeaux.fr

3 Université de Savoie - Polytech Annecy-Chambéry, LISTIC, Philippe.Bolon@univ-savoie.fr
}

that a large amount of frequencies contributes in the corresponding wavelet variances.

The present paper investigates the capability of 2-D wavelet packets to yield an accurate Power Spectral Density (PSD) estimator for characterizing second order statistical properties of random fields. In this paper, we consider the standard Fourier spectrum as benchmark and seek for estimating this spectrum by using suitable wavelets. This positions the Shannon wavelet at the focus of the paper: as the decomposition level tends to infinity, the bias of the Shannon wavelet spectrum estimator tends to 0 . The bias of an arbitrary wavelet spectrum estimator then relates to the closeness of the wavelet under consideration to the Shannon wavelet. This closeness is measured through a parameter called wavelet order.

The results derived in the paper are in continuation of [5] which introduces PSD estimation by using the statistical properties of the wavelet packet coefficients of random processes. The paper focuses on presenting the specificities that follow by dimensionality increasing from [5]:

- the analytic form of the wavelet packet based PSD,

- the singular paths of fractional Brownian fields (Section III),

- the 2-D wavelet packet PSD estimator (Section IV).

Furthermore, for textured image analysis, the paper provides experimental results for evaluating the relevance of spectrum estimation by 1) comparing PSD estimated from Fourier and wavelet packet methods and 2) performing content based image retrieval associated with spectral similarity measurements in the Fourier and wavelet packet domains (Section V).

\section{2-D WAVELET PACKETS}

This section provides a brief introduction to 2-D wavelet packet transforms. Advanced concepts and algorithms concerning $1 \mathrm{D}$ and 2-D wavelet packet analysis can be found in [6], [7], [8]. The reader is also invited to refer to [9], [10], [11], [12], [13] (wavelets) and [5], [14] (wavelet packets) for more details on the statistical properties of wavelet transforms, when the decomposition relates to a random process.

\section{A. Introduction on 2-D wavelet packets}

We consider the 2-D separable wavelet packet decomposition in a continuous time signal setting for presenting theoretical results (see [7] for the connection between the continuous and the discrete wavelet transforms). In this decomposition, the wavelet paraunitary filters $H_{0}$ (low-pass, 
scaling filter) and $H_{1}$ (high-pass, wavelet filter) are used to split the input functional space $\mathbf{U}=\mathbf{W}_{0,0} \subset L^{2}\left(\mathbb{R}^{2}\right)$ into orthogonal subspaces (subbands):

$$
\begin{aligned}
\mathbf{W}_{0,0}= & \mathbf{W}_{1,[0,0]} \oplus \mathbf{W}_{1,[1,0]} \oplus \mathbf{W}_{1,[0,1]} \oplus \mathbf{W}_{1,[1,1]}, \\
\mathbf{W}_{j,\left[n_{1}, n_{2}\right]}= & \mathbf{W}_{j+1,\left[2 n_{1}, 2 n_{2}\right]} \oplus \mathbf{W}_{j+1,\left[2 n_{1}+1,2 n_{2}\right]} \oplus \ldots \\
& \oplus \mathbf{W}_{j+1,\left[2 n_{1}, 2 n_{2}+1\right]} \oplus \mathbf{W}_{j+1,\left[2 n_{1}+1,2 n_{2}+1\right]}
\end{aligned}
$$

for $j \geqslant 1$. The subbands involved in this quad-tree splitting trick follow from a separable product $\mathbf{W}_{j,\left[n_{1}, n_{2}\right]}=\mathbf{W}_{j, n_{1}} \otimes$ $\mathbf{W}_{j, n_{2}}$ of 1D wavelet packet subspaces $\mathbf{W}_{j, n_{1}}$ and $\mathbf{W}_{j, n_{2}}$. We assume that $\mathbf{U}$ is the subspace spanned by an orthonormal system $\left\{\tau_{\left[k_{1}, k_{2}\right]} \Phi:\left(k_{1}, k_{2}\right) \in \mathbb{Z}^{2}\right\}$ with $\Phi \in L^{2}\left(\mathbb{R}^{2}\right)$ and where $\tau$ is the shift operator: $\tau_{\left[k_{1}, k_{2}\right]} f\left(t_{1}, t_{2}\right)=f\left(t_{1}-k_{1}, t_{2}-k_{2}\right)$.

Denote $\mathscr{F} f$, the Fourier transform of $f \in L^{1}\left(\mathbb{R}^{2}\right) \cup L^{2}\left(\mathbb{R}^{2}\right)$, with

$$
\mathscr{F} f\left(\omega_{1}, \omega_{2}\right)=\int_{\mathbb{R}^{2}} f\left(t_{1}, t_{2}\right) e^{-i\left(\omega_{1} t_{1}+\omega_{2} t_{2}\right)} \mathrm{d} t_{1} \mathrm{~d} t_{2} .
$$

Since $\mathbf{U}$ does not necessarily depend on the wavelet filters, we assume in the following that $\mathbf{U}$ is the space of functions having their Fourier transforms with supports in $[-\pi, \pi] \times$ $[-\pi, \pi]$. In this respect, $\Phi$ is the $\operatorname{sinc}$ function:

$$
\Phi\left(t_{1}, t_{2}\right)=\operatorname{sinc}\left(t_{1}, t_{2}\right)=\frac{\sin \left(\pi t_{1}\right) \sin \left(\pi t_{2}\right)}{\pi^{2} t_{1} t_{2}} .
$$

The wavelet packet subband $\mathbf{W}_{j,\left[n_{1}, n_{2}\right]}$ is, by construction, the closure of the $L^{2}\left(\mathbb{R}^{2}\right)$ subspace spanned by the orthornormal system of wavelet packet functions:

$$
\left\{\tau_{\left[2^{j} k_{1}, 2^{j} k_{2}\right]} W_{j,\left[n_{1}, n_{2}\right]}:\left(k_{1}, k_{2}\right) \in \mathbb{Z}^{2}\right\} .
$$

Due to separability, the wavelet packet function $W_{j,\left[n_{1}, n_{2}\right]}$ satisfies:

$$
\mathscr{F} W_{j,\left[n_{1}, n_{2}\right]}\left(\omega_{1}, \omega_{2}\right)=\mathscr{F} W_{j, n_{1}}\left(\omega_{1}\right) \mathscr{F} W_{j, n_{2}}\left(\omega_{2}\right) .
$$

By decomposing the frequency indices $n_{1}, n_{2}$ in the form

$$
n_{1}=\sum_{\ell=1}^{j} \epsilon_{\ell}^{1} 2^{j-\ell}, \quad n_{2}=\sum_{\ell=1}^{j} \epsilon_{\ell}^{2} 2^{j-\ell},
$$

with $\left(\epsilon_{\ell}^{1}, \epsilon_{\ell}^{2}\right) \in\{0,1\}^{2}$ for every $\ell \in\{1,2, \ldots, j\}$, we have

$$
\mathscr{F} W_{j,\left[n_{1}, n_{2}\right]}=\mathbf{H}_{j,\left[n_{1}, n_{2}\right]} \mathscr{F} \Phi,
$$

where the multiscale wavelet packet filter $\mathbf{H}_{j,\left[n_{1}, n_{2}\right]}$ applied to obtain the subband $(j, n)$ wavelet packet coefficients is

$$
\mathbf{H}_{j,\left[n_{1}, n_{2}\right]}\left(\omega_{1}, \omega_{2}\right)=\prod_{i=1}^{2} \mathbf{H}_{j, n_{i}}\left(\omega_{i}\right)
$$

with

$$
\mathbf{H}_{j, n_{i}}(\omega)=2^{j / 2}\left[\prod_{\ell=1}^{j} H_{\epsilon_{\ell}^{i}}\left(2^{\ell-1} \omega\right)\right]
$$

for $\epsilon_{\ell}^{i} \in\{0,1\}, i=1,2$, where $H_{0}$ and $H_{1}$ are the scaling and the wavelet filter introduced above.

Assume that the scaling filter is with order $r: H_{0} \equiv H_{0}^{r}$, where $r$ is the largest non-negative integer such that [15]

$$
H_{0}^{r}(\omega)=\left(\frac{1+e^{-i \omega}}{2}\right)^{r} Q\left(e^{i \omega}\right)
$$

and $Q$ is a polynomial, with the following equality holding almost everywhere (a.e.):

$$
\lim _{r \rightarrow \infty} H_{0}^{r}(\omega) \stackrel{\text { a.e. }}{=} H_{0}^{\mathrm{S}}(\omega),
$$

filter $H_{0}^{\mathrm{S}}$ denoting the scaling filter associated with the Shannon wavelet. Then the 1D multiscale filters $\left(\mathbf{H}_{j, n_{i}}^{r}\right)_{i=1,2}$ have very tight supports when $r$ is large (see [14, Figure 1] for illustration). These multiscale filters tend to the Shannon ideal filters $\mathbf{H}_{j,\left[n_{1}, n_{2}\right]}^{\mathrm{S}}$ when $r$ increases (see Eq. (4) and Eq. (6)). The consequence is that the spectrum of the input random field is analyzed within a frequency window with small size. Indeed, the restriction of the support of $\mathbf{H}_{j,\left[n_{1}, n_{2}\right]}^{\mathrm{S}}$ to $[-\pi, \pi] \times[-\pi, \pi]$ is with size $2^{-j} \times 2^{-j}$. This makes possible a local analysis of the spectrum, as performed in Section IV below.

\section{B. Wavelet packet paths}

This section presents a specific wavelet packet path description derived from the binary sequence approach of [16] for representing nested wavelet packet subspaces. This description is suitable for establishing asymptotic properties of 2-D wavelet packets with respect to the increase of the decomposition level.

For the sake of simplifying notation, 2-D wavelet packets are commonly presented through a quad-tree structure where a pair $\left[n_{1}, n_{2}\right] \in\left\{0,1, \ldots, 2^{j}-1\right\}^{2}$ of frequency indices is set in a one to one correspondence with an index $n \in\left\{0,1, \ldots, 4^{j}-1\right\}$. This correspondence leads to a single frequency indexing of the wavelet packet subbands.

More precisely, this correspondence follows by associating a quaternary index $\mu \in\{0,1,2,3\}$

$$
\mu=2 \epsilon^{1}+\epsilon^{2}=\left\{\begin{array}{lll}
0 & \text { if } & \left(\epsilon^{1}, \epsilon^{2}\right)=(0,0) \\
1 & \text { if } & \left(\epsilon^{1}, \epsilon^{2}\right)=(0,1) \\
2 & \text { if } & \left(\epsilon^{1}, \epsilon^{2}\right)=(1,0) \\
3 & \text { if } & \left(\epsilon^{1}, \epsilon^{2}\right)=(1,1)
\end{array}\right.
$$

to the binary indices $\left(\epsilon^{1}, \epsilon^{2}\right) \in\{0,1\}^{2}$ and by defining:

$$
n=n(j)=\sum_{\ell=1}^{j} \mu_{\ell} 4^{j-\ell} .
$$

The above change of variable is a univoque correspondence between $\left(n_{1}, n_{2}\right) \in\left\{0,1, \ldots, 2^{j}-1\right\}^{2}$ defined by Eq. (2) and $n \in\left\{0,1, \ldots, 4^{j}-1\right\}$ defined by Eq. (8). This correspondence makes it possible to characterize the 4-band tree structure of the 2-D wavelet packet decomposition with a single shift parameter $n$, instead of the more intricate tree description with double indexed frequency indices.

This characterization also makes it possible to use a straightforward path based description of the wavelet packet tree by associating an infinite quaternary sequence $\bar{\mu}=\left(\mu_{1}, \mu_{2}, \mu_{3}, \ldots\right)$ to a wavelet packet path $\mathscr{P} \equiv \mathscr{P}_{\bar{\mu}}$ and vice versa or, equivalently, an infinite sequence of frequency indices $\bar{n}=(n(1), n(2), n(3), \ldots)$, those associated with $\bar{\mu}$ to $\mathscr{P} \equiv \mathscr{P}_{\bar{n}}$ from Eq. (8).

Let $\left(\epsilon_{k}^{1}\right)_{k \in \mathbb{N}}$ and $\left(\epsilon_{k}^{2}\right)_{k \in \mathbb{N}}$ be the binary sequences associated with $\bar{\mu}$ from the correspondence given by Eq. 
(7). Then, by taking into account Eq. (2), frequency index $n_{\mathscr{P}}(j)$ is also associated with a pair of frequency indices $\left(n_{1}[\mathscr{P}](j), n_{2}[\mathscr{P}](j)\right)$.

In the rest of the paper, we will use notation $n, n_{\mathscr{P}}(j)$ in general whereas notation $n_{1}, n_{2}, n_{1}[\mathscr{P}](j), n_{2}[\mathscr{P}](j)$ will be used to specify the spatial location of the frequency support of the wavelet packet subband under consideration.

To end this section, it is worth mentioning that some specific paths will present singular behavior, depending on the input random field: the wavelet coefficients of certain non-stationary random fields on the subbands associated with these singular paths will remain non-stationary. As a matter of example,

- The separable fractional Brownian field analyzed in Section III-B1 admits frequency indices $n(j)$ such that $n_{1}(j)=0$ (resp. $n_{2}(j)=0$ ) for every $j$ as singular frequency indices. The set of (singular) paths associated with these frequency indices will be denoted by $\mathscr{P}_{\bar{n} / \bar{n}_{1}=0}$ (resp. $\mathscr{P}_{\bar{n} / \bar{n}_{2}=0}$ ).

- The isotropic fractional Brownian field analyzed in Section III-B2 admits a unique singular path: the approximation path denoted by $\mathscr{P}_{0}$ and associated with frequency indices $n_{\mathscr{P}_{0}}(j)=0$ for every $j$.

\section{2-D WAVELET PACKETS OF SECOND ORDER RANDOM FIELDS}

\section{A. The case of stationary random fields}

Let $X=X\left(t_{1}, t_{2}\right)$ be a second-order zero-mean real valued WSS random field. We assume that $X$ is continuous in quadratic mean.

The autocorrelation function of $X$ is $R\left(t_{1}, t_{2}, s_{1}, s_{2}\right)=$ $\mathbb{E}\left[X\left(t_{1}, t_{2}\right) X\left(s_{1}, s_{2}\right)\right]$. We assume that $R$ satisfies

$$
\begin{aligned}
& \int_{\mathbb{R}^{4}} R\left(t_{1}, t_{2}, s_{1}, s_{2}\right) \tau_{\left[2^{j} k_{1}, 2^{j} k_{2}\right]} W_{j, n}\left(t_{1}, t_{2}\right) \\
& \tau_{\left[2^{j} k_{1}, 2^{j} k_{2}\right]} W_{j, n}\left(s_{1}, s_{2}\right) \mathrm{d} t_{1} \mathrm{~d} t_{2} \mathrm{~d} s_{1} \mathrm{~d} s_{2}<\infty .
\end{aligned}
$$

Under WSS assumption, this autocorrelation function reduces to a two variable function by using the convention:

$$
R\left(t_{1}, t_{2}, s_{1}, s_{2}\right) \equiv R\left(t_{1}-s_{1}, t_{2}-s_{2}\right)=R\left(\rho_{1}, \rho_{2}\right)
$$

where $\rho_{i}=t_{i}-s_{i}$ for $i=1,2$.

We assume that $\gamma=\mathscr{F} R$ exists,

$$
\gamma\left(\omega_{1}, \omega_{2}\right)=\int_{\mathbb{R}^{2}} R\left(\rho_{1}, \rho_{2}\right) e^{-i\left(\omega_{1} \rho_{1}+\omega_{2} \rho_{2}\right)} \mathrm{d} \rho_{1} \mathrm{~d} \rho_{2} .
$$

Function $\gamma$ is the PSD of the WSS random field $X$.

Under assumptions mentioned above, the coefficients of $X$ on subband $\mathbf{W}_{j, n}$ define a discrete second order random field

$$
c_{j, n}\left[k_{1}, k_{2}\right]=\int_{\mathbb{R}^{2}} X\left(t_{1}, t_{2}\right) \tau_{2^{j}\left[k_{1}, k_{2}\right]} W_{j, n}\left(t_{1}, t_{2}\right) \mathrm{d} t_{1} \mathrm{~d} t_{2} .
$$

Random field $c_{j, n}$ inherits the WSS property of $X$ : the autocorrelation function $R_{j, n}$ of $c_{j, n}$,

$$
\begin{array}{r}
R_{j, n}\left[k_{1}, k_{2}, \ell_{1}, \ell_{2}\right]=\int_{\mathbb{R}^{4}} R\left(t_{1}, t_{2}, s_{1}, s_{2}\right) \tau_{2^{j}\left[k_{1}, k_{2}\right]} W_{j, n}\left(t_{1}, t_{2}\right) \\
\tau_{2^{j}\left[\ell_{1}, \ell_{2}\right]} W_{j, n}\left(s_{1}, s_{2}\right) \mathrm{d} t_{1} \mathrm{~d} t_{2} \mathrm{~d} s_{1} \mathrm{~d} s_{2}
\end{array}
$$

reduces to $R_{j, n}\left[k_{1}, k_{2}, \ell_{1}, \ell_{2}\right]=R_{j, n}\left[k_{1}-\ell_{1}, k_{2}-\ell_{2}\right]=$ $R_{j, n}\left[m_{1}, m_{2}\right]$. Moreover, if we assume that PSD $\gamma$ of $X$ is bounded on the support of $\mathscr{F} W_{j, n}$, then function $R_{j, n}\left[m_{1}, m_{2}\right]$ has the form

$$
\begin{array}{r}
R_{j, n}\left[m_{1}, m_{2}\right]=\frac{1}{4 \pi^{2}} \int_{\mathbb{R}^{2}} \gamma\left(\omega_{1}, \omega_{2}\right)\left|\mathscr{F} W_{j, n}\left(\omega_{1}, \omega_{2}\right)\right|^{2} \\
e^{i 2^{j}\left(m_{1} \omega_{1}+m_{2} \omega_{2}\right)} \mathrm{d} \omega_{1} \mathrm{~d} \omega_{2} .
\end{array}
$$

B. The case of non-stationary random fields: application to separable and isotropic fractional Brownian fields

Associating a wavelet packet based PSD to a non-WSS random field involves finding the WSS subbands (if any) associated with the decomposition of this field. Specifically, we consider in this section, the wavelet packet decompositions of some separable and isotropic constructions of random fields from the $1 \mathrm{D}$ fractional Brownian motion. Such a random field is called a fractional Brownian field (fBf).

1) Separable fractional Brownian field: This $\mathrm{fBf}$, hereafter denoted by $X_{\alpha}^{\mathfrak{s}}=X_{\alpha}^{\mathfrak{s}}\left(t_{1}, t_{2}\right)$, where $\alpha$ is the Hurst parameter, follows from a separable 2-D extension (cartesian product) of a 1D fractional Brownian motion. Its autocorrelation function is

$$
\begin{aligned}
R^{\mathfrak{s}}\left(t_{1}, t_{2}, s_{1}, s_{2}\right)=\frac{\sigma^{2}}{4} & \left(\left|t_{1}\right|^{2 \alpha}+\left|s_{1}\right|^{2 \alpha}-\left|t_{1}-s_{1}\right|^{2 \alpha}\right) \\
& \times\left(\left|t_{2}\right|^{2 \alpha}+\left|s_{2}\right|^{2 \alpha}-\left|t_{2}-s_{2}\right|^{2 \alpha}\right)
\end{aligned}
$$

where $\sigma$ is a constant that can be tuned so as to control the variance of the $\mathrm{fBf}$ at a specific spatial coordinates.

Assume that the wavelet function $W_{j, n}=W_{j, n}\left(t_{1}, t_{2}\right)$ is with compact support and has (at least) one vanishingmoment in each variable $t_{1}, t_{2}$. This assumption is satisfied for the frequency indices $n$ such that $n_{1} \neq 0$ and $n_{2} \neq 0$. The corresponding subbands $\left(4^{j}-2^{j+1}+1\right.$ subbands at decomposition level $j$ ) are hereafter called detail wavelet packet subbands since their generating functions have vanishing moments in each direction.

The following Proposition 1 thus concerns the $4^{j}-2^{j+1}+1$ detail wavelet packet subbands. In these subbands, the wavelet packet moments vanish and the autocorrelation function $R_{j, n}^{\mathfrak{s}}$ of $c_{j, n}^{\mathfrak{s}}$ reduces to

$$
\begin{aligned}
& R_{j, n}^{\mathfrak{s}}\left[k_{1}, k_{2}, \ell_{1}, \ell_{2}\right]= \\
& \quad \frac{\sigma^{2}}{4} \int_{\mathbb{R}^{4}}\left|t_{1}-s_{1}\right|^{2 \alpha}\left|t_{2}-s_{2}\right|^{2 \alpha} \tau_{\left[2^{j} k_{1}, 2^{j} k_{2}\right]} W_{j, n}\left(t_{1}, t_{2}\right) \\
& \quad \tau_{\left[2^{j} \ell_{1} 2^{j} \ell_{2}\right]} W_{j, n}\left(s_{1}, s_{2}\right) \mathrm{d} t_{1} \mathrm{~d} t_{2} \mathrm{~d} s_{1} \mathrm{~d} s_{2} .
\end{aligned}
$$

Assume furthermore that function $W_{j, n}$ is with compact support or has sufficiently fast decay, and that the Fourier transform of $W_{j, n}$ has a bounded derivative in the neighborhood of the origin, then we can state the following Proposition 1.

Proposition 1: The discrete random field $c_{j, n}^{\mathfrak{s}}$ representing the coefficients of $X_{\alpha}^{\mathfrak{s}}$ on $\mathbf{W}_{j, n}$ is WSS when the frequency index $n$ is such that $n_{1} \neq 0$ and $n_{2} \neq 0$. Its 
autocorrelation function $R_{j, n}^{\mathfrak{s}}\left[k_{1}, k_{2}, \ell_{1}, \ell_{2}\right]=R_{j, n}^{\mathfrak{s}}\left[k_{1}-\ell_{1}, k_{2}-\right.$ $\left.\ell_{2}\right]=R_{j, n}^{\mathfrak{s}}\left[m_{1}, m_{2}\right]$, is:

$$
\begin{aligned}
& R_{j, n}^{\mathfrak{s}}\left[m_{1}, m_{2}\right]= \\
& \frac{1}{4 \pi^{2}} \int_{\mathbb{R}^{2}} \gamma^{\mathfrak{s}}\left(\omega_{1}, \omega_{2}\right)\left|\mathscr{F} W_{j, n}\left(\omega_{1}, \omega_{2}\right)\right|^{2} \\
& e^{i 2^{j}\left(m_{1} \omega_{1}+m_{2} \omega_{2}\right)} \mathrm{d} \omega_{1} \mathrm{~d} \omega_{2},
\end{aligned}
$$

where

$$
\gamma^{\mathfrak{s}}\left(\omega_{1}, \omega_{2}\right)=\frac{\sigma^{2} \Gamma^{2}(2 \alpha+1) \sin ^{2}(\pi \alpha)}{\left|\omega_{1}\right|^{2 \alpha+1} \times\left|\omega_{2}\right|^{2 \alpha+1}}
$$

and the Gamma function is defined by $\Gamma(u)=$ $\int_{\mathbb{R}^{+}} x^{u-1} e^{-x} \mathrm{~d} x$.

From Proposition 1, we derive that stationarity holds true for the $4^{j}-2^{j+1}+1$ detail wavelet subbands. Let us focus on the frequency axes, that is when $n_{1}=0$ and $n_{2} \neq 0$, or vice versa. For such an index $n$, we have

$$
\begin{gathered}
R_{j, n}^{\mathfrak{s}}\left[k_{1}, k_{2}, \ell_{1}, \ell_{2}\right]=\Lambda_{j, n}^{\mathfrak{s}}\left[k_{1}, k_{2}, \ell_{1}, \ell_{2}\right] \\
+\frac{1}{4 \pi^{2}} \int_{\mathbb{R}^{2}} \gamma^{\mathfrak{s}}\left(\omega_{1}, \omega_{2}\right)\left|\mathscr{F} W_{j, n}\left(\omega_{1}, \omega_{2}\right)\right|^{2} \\
e^{i 2^{j}\left(m_{1} \omega_{1}+m_{2} \omega_{2}\right)} \mathrm{d} \omega_{1} \mathrm{~d} \omega_{2}
\end{gathered}
$$

where, as above, $m_{i}=k_{i}-\ell_{i}$ for $i=1,2$ and

$\Lambda_{j, n}^{\mathfrak{s}}\left[k_{1}, k_{2}, \ell_{1}, \ell_{2}\right]=\left\{\begin{array}{lll}\mathscr{M}_{j, 0, k_{2}}^{2 \alpha} \times{ }_{2} R_{j, n_{2}}^{\mathfrak{s}}\left[m_{2}\right] & \text { if } & n_{1}=0, n_{2} \neq 0 \\ \mathscr{M}_{j, 0, \ell_{1}}^{2 \alpha} \times{ }_{1} R_{j, n_{1}}^{\mathfrak{s}}\left[m_{1}\right] & \text { if } & n_{2}=0, n_{1} \neq 0\end{array}\right.$ and $\mathscr{M}_{j, n_{i}, k_{i}}^{2 \alpha}$ is the $2 \alpha$ fractional moment of the $1 \mathrm{D}$ wavelet function $\tau_{2^{j} k_{i}} W_{j, n_{i}}$ :

$$
\mathscr{M}_{j, 0, k_{i}}^{2 \alpha}=\int_{\mathbb{R}}|t|^{2 \alpha} \tau_{2^{j} k_{i}} W_{j, 0}(t) \mathrm{d} t,
$$

with ${ }_{1} R_{j, n_{1}}^{\mathfrak{s}}$ (resp. ${ }_{2} R_{j, n_{2}}^{\mathfrak{s}}$ ) denoting the autocorrelation function of a $1 \mathrm{D}$ section of an $\mathrm{fBf}$ (thus a fractional Brownian motion),

$$
{ }_{1} R_{j, n_{1}}^{\mathfrak{s}}\left[m_{1}\right]=\frac{1}{2 \pi} \int_{\mathbb{R}} \gamma^{*}\left(\omega_{1}\right)\left|\mathscr{F} W_{j, n_{1}}\left(\omega_{1}\right)\right|^{2} e^{i 2^{j} m_{1} \omega_{1}} \mathrm{~d} \omega_{1}
$$

when $n_{1} \neq 0$ and

$$
{ }_{2} R_{j, n_{2}}^{\mathfrak{s}}\left[m_{2}\right]=\frac{1}{2 \pi} \int_{\mathbb{R}} \gamma^{*}\left(\omega_{2}\right)\left|\mathscr{F} W_{j, n_{2}}\left(\omega_{2}\right)\right|^{2} e^{i 2^{j} m_{2} \omega_{2}} \mathrm{~d} \omega_{2},
$$

when $n_{2} \neq 0$. In these equations, function $\gamma^{*}$ is given by

$$
\gamma^{*}(\omega)=\frac{\sigma^{2} \Gamma(2 \alpha+1) \sin (\pi \alpha)}{|\omega|^{2 \alpha+1}} .
$$

One can notice the presence of an additive nonstationary term $\Lambda_{j, n}^{\mathfrak{s}}\left[k_{1}, k_{2}, \ell_{1}, \ell_{2}\right]$ in Eq. (16). This term is a $2 \alpha$ centered fractional moment of the scaling function $W_{j, 0}$. This fractional moment is centered at $-2^{j} k_{2}$ when $n_{1}=0$ (resp. at $-2^{j} \ell_{1}$ when $n_{2}=0$ ). The height of this fractional moment depends on the scaling function associated with the wavelet packet decomposition.

Note also that when both $n_{1}=n_{2}=0$, the nonstationarity in wavelet coefficients is more intricate, mainly because the analyzing function has no vanishing moments in neither of the variables $t_{1}, t_{2}$.
2) Isotropic fractional Brownian field: This random field, denoted by $X_{\alpha}^{\mathfrak{i}}=X_{\alpha}^{\mathfrak{i}}\left(t_{1}, t_{2}\right)$ is such that any of its $1 \mathrm{D}$ section by a line segment issued from the origin is a fractional Brownian motion. Its autocorrelation function is defined by

$$
\begin{aligned}
R^{\mathrm{i}}\left(t_{1}, t_{2}, s_{1}, s_{2}\right)= & \frac{\sigma^{2}}{2}\left(\left(t_{1}^{2}+t_{2}^{2}\right)^{\alpha}+\left(s_{1}^{2}+s_{2}^{2}\right)^{\alpha}\right) \\
& -\frac{\sigma^{2}}{2}\left[\left(t_{1}-s_{1}\right)^{2}+\left(t_{2}-s_{2}\right)^{2}\right]^{\alpha} .
\end{aligned}
$$

By following a reasoning analog to that of Section III-B1 above, we have

$$
\begin{aligned}
& R_{j, n}^{\mathrm{i}}\left[k_{1}, k_{2}, \ell_{1}, \ell_{2}\right]= \\
& -\frac{\sigma^{2}}{2} \int_{\mathbb{R}^{4}}\left[\left(t_{1}-s_{1}\right)^{2}+\left(t_{2}-s_{2}\right)^{2}\right]^{\alpha} \tau_{\left[2^{j} k_{1}, 2^{j} k_{2}\right]} W_{j, n}\left(t_{1}, t_{2}\right) \\
& \tau_{\left[2^{j} \ell_{1}, 2^{j} \ell_{2}\right]} W_{j, n}\left(s_{1}, s_{2}\right) \mathrm{d} t_{1} \mathrm{~d} t_{2} \mathrm{~d} s_{1} \mathrm{~d} s_{2}
\end{aligned}
$$

under integrability condition for the term $\left(t_{1}^{2}+t_{2}^{2}\right)^{\alpha} W_{j, n}\left(t_{1}, t_{2}\right)$ (compact support or sufficiently fast decay for $W_{j, n}$ ) and provided that $n \neq 0$ (the wavelet function $W_{j, n}$ then have at least one vanishing moment in some direction).

Furthermore, if $\mathscr{F} W_{j, n}\left(\omega_{1}, \omega_{2}\right) /\left(\omega_{1}^{2}+\omega_{2}^{2}\right)^{3 / 4}$ is bounded in the neighborhood of the origin, then we have the following result:

Proposition 2: The random field $c_{j, n}^{\mathrm{i}}=$ $\left(c_{j, n}^{\mathfrak{i}}\left[k_{1}, k_{2}\right]\right)_{\left[k_{1}, k_{2}\right] \in \mathbb{Z} \times \mathbb{Z}}$ representing subband $\mathbf{W}_{j, n}$ coefficients of $X_{\alpha}^{\mathrm{i}}$ is WSS for $n \neq 0$, with autocorrelation function $R_{j, n}^{\mathrm{i}}\left[k_{1}, k_{2}, \ell_{1}, \ell_{2}\right] \equiv R_{j, n}^{\mathrm{i}}\left[k_{1}-\ell_{1}, k_{2}-\ell_{2}\right]=R_{j, n}^{\mathrm{i}}\left[m_{1}, m_{2}\right]$ given by:

$$
\begin{array}{r}
R_{j, n}^{\mathrm{i}}\left[m_{1}, m_{2}\right]=\frac{1}{4 \pi^{2}} \int_{\mathbb{R}^{2}} \gamma^{\mathrm{i}}\left(\omega_{1}, \omega_{2}\right)\left|\mathscr{F} W_{j, n}\left(\omega_{1}, \omega_{2}\right)\right|^{2} \\
e^{i 2^{j}\left(m_{1} \omega_{1}+m_{2} \omega_{2}\right)} \mathrm{d} \omega_{1} \mathrm{~d} \omega_{2},
\end{array}
$$

where

$$
\gamma^{\mathfrak{i}}\left(\omega_{1}, \omega_{2}\right)=\frac{2^{-(2 \alpha+1)} \pi^{2} \sigma^{2}}{\sin (\pi \alpha) \Gamma^{2}(1+\alpha)} \frac{1}{\left(\omega_{1}^{2}+\omega_{2}^{2}\right)^{\alpha+1}} .
$$

From Propositions 1 and 2, it follows that:

Remark 1: Except for a few frequency indices given in Propositions 1 and 2, the autocorrelation function of the wavelet packet coefficients of separable and isotropic $\mathrm{fBfs}$ can be written in the integral form involved in Eq. (12).

From now on, when no confusion is possible, the autocorrelation close form given by Eq. (12) will also be used to denote the autocorrelation function of separable fBf (see Eq. (14)) or isotropic fBf (see Eq. (20)).

More precisely, when the autocorrelation function of the wavelet packet coefficients of an arbitrary, non-stationary random field can be written in the form given by Eq. (12) for a specific function $\gamma$, this function will be called the spectrum of the random field under consideration.

The following section addresses the estimation of spectrum $\gamma$. The method proposed relies on the frequency partitioning induced by the Shannon wavelet packets. 


\section{2-D WAVELET PACKET BASED SPECTRUM ESTIMATION}

The spectrum estimation method presented in this section follows from the asymptotic analysis of the autocorrelation functions of the 2-D wavelet packet coefficients. This asymptotic analysis is performed with respect to the wavelet order $r$ and the wavelet decomposition level $j$. When $r$ increases, the asymptotic behavior of the sequence of wavelet functions is driven by the Shannon wavelet functions (see Eq. (6)). In this respect, we consider the Shannon wavelets in Section IV-A below and derive asymptotic results with respect to the wavelet decomposition level. The case of a wavelet with order $r$ will be considered as an approximation of the Shannon limiting behavior when $r$ is large enough.

\section{A. Autocorrelation functions of the Shannon wavelet packets and asymptotic analysis}

When the Shannon ideal paraunitary filters are used, the Fourier transform of the Shannon wavelet packet function $W_{j, n}^{\mathrm{S}}$ satisfies:

$$
\left|\mathscr{F} W_{j, n}^{S}\left(\omega_{1}, \omega_{2}\right)\right|=2^{j} \mathbb{1}_{\Delta_{j, G\left(n_{1}\right)} \times \Delta_{j, G\left(n_{2}\right)}}\left(\omega_{1}, \omega_{2}\right),
$$

where $\mathbb{1}_{K}$ denotes the indicator function of the set $K$ and $\Delta_{j, G(n)}=\Delta_{j, G(n)}^{-} \cup \Delta_{j, G(n)}^{+}$, with $\Delta_{j, G(n)}^{-}=-\Delta_{j, G(n)}^{+}$,

$$
\Delta_{j, G(n)}^{+}=\left[\frac{G(n) \pi}{2^{j}}, \frac{(G(n)+1) \pi}{2^{j}}\right] .
$$

The function $G$ in Eq. (23) is a permutation (see [6], [7] for details) defined by $G(0)=0$ and

$$
G(2 \ell+\epsilon)=3 G(\ell)+\epsilon-2\left\lfloor\frac{G(\ell)+\epsilon}{2}\right\rfloor,
$$

where $\lfloor z\rfloor$ is the largest integer less than or equal to $z$ and $\epsilon \in\{0,1\}$.

From Eqs. (12), (15), (21), (22), the autocorrelation functions of the Shannon wavelet packet coefficients of the WSS random field described above, the separable fBf (with $n_{1} \neq 0$ and $n_{2} \neq 0$ when separable fBf is considered) and the isotropic $\mathrm{fBf}$ (assumes $\left(n_{1}, n_{2}\right) \neq 0$ for the isotropic fBf) can be written in the form

$$
\begin{gathered}
R_{j, n}^{\mathrm{S}}\left[m_{1}, m_{2}\right]=\frac{2^{2 j}}{\pi^{2}} \int_{\Delta_{j, G\left(n_{1}\right)}^{+} \times \Delta_{j, G\left(n_{2}\right)}^{+}} \cos \left(2^{j} m_{1} \omega_{1}\right) \cos \left(2^{j} m_{2} \omega_{2}\right) \\
\gamma\left(\omega_{1}, \omega_{2}\right) \mathrm{d} \omega_{1} \mathrm{~d} \omega_{2}
\end{gathered}
$$

where $\gamma$ is the spectrum of the input random field: $\gamma$ is given by Eq. (9) for a WSS random field and $\gamma$ is given by Eq. (15) (resp. Eq. (21)) for the separable (resp. isotropic) fBf.

The asymptotic analysis of these autocorrelations when $j$ tends to infinity is performed by considering a path $\mathscr{P}$ of the 2-D wavelet packet decomposition tree. We assume that

- $\mathscr{P} \neq \mathscr{P}_{0}$ in the case of the isotropic fBf and

- $\mathscr{P} \notin\left\{\mathscr{P}_{\bar{n} / \bar{n}_{1}=0} \cup \mathscr{P}_{\bar{n} / \bar{n}_{2}=0}\right\}$ in the case of the separable fBf.

Thus, the sequence of autocorrelation functions $\left(R_{j, n_{\mathscr{P}}(j)}^{\mathrm{S}}\right)_{j \geqslant 1}$ of Shannon wavelet packets in path $\mathscr{P}$ is such that every $R_{j, n_{\mathscr{P}}(j)}^{S}$, for $j \geqslant 1$, has the form given by Eq. (25).

Let

$$
\begin{aligned}
& \omega_{1}[\mathscr{P}]=\lim _{j \rightarrow+\infty} \frac{G\left(n_{1}[\mathscr{P}](j)\right) \pi}{2^{j}}, \\
& \omega_{2}[\mathscr{P}]=\lim _{j \rightarrow+\infty} \frac{G\left(n_{2}[\mathscr{P}](j)\right) \pi}{2^{j}} .
\end{aligned}
$$

Assume that $\left(\omega_{1}[\mathscr{P}], \omega_{2}[\mathscr{P}]\right)$ is a continuity point of the spectrum $\gamma$. Then, the sequence of autocorrelation functions $\left(R_{j, n_{\mathscr{P}}(j)}^{S}\right)$ satisfies:

$$
\lim _{j \rightarrow+\infty} R_{j, n}^{\mathrm{S}}\left[m_{1}, m_{2}\right]=\gamma\left(\omega_{1}[\mathscr{P}], \omega_{2}[\mathscr{P}]\right) \delta\left[m_{1}, m_{2}\right]
$$

uniformly in $\left(m_{1}, m_{2}\right) \in \mathbb{Z}^{2}$, where $\delta[\cdot, \cdot]$ is defined by

$$
\delta\left[k_{1}, k_{2}\right]=\left\{\begin{array}{lll}
1 & \text { if } & k_{1}=k_{2}=0, \\
0 & \text { otherwise. }
\end{array}\right.
$$

\section{B. 2-D Wavelet packet based spectrum estimation}

The following provides a non-parametric method for estimating spectrum $\gamma$ of 2-D random fields on the basis of the convergence criteria given by Eqs. (6) and (28).

From Eq. (28), it follows that $\gamma\left(\omega_{1}[\mathscr{P}], \omega_{2}[\mathscr{P}]\right)=$ $\lim _{j \rightarrow+\infty} R_{j, n}^{S}[0,0]$ so that the continuity points of spectrum $\gamma$ can be estimated by subband variances (values $\left.\left\{R_{j, n}^{S}[0,0]\right\}_{n}\right)$, provided that the Shannon wavelet is used and $j$ is large enough. Furthermore, we can derive from the convergence criteria given by Eq. (6), several spectrum estimators by considering wavelets with finite orders $r$ (Shannon wavelet corresponds to $r=+\infty \equiv \mathrm{S}$ ), the accuracy of the spectrum estimation being dependent on the wavelet order as shown in Proposition 3 below.

Assuming a uniform sampling (regularly spaced frequency plane tiling), the method applies upon the following steps.

\section{2-D Wavelet Packet Spectrum}

1) Define a frequency grid composed with frequency points $\left(\frac{p_{1} \pi}{2^{j}}, \frac{p_{2} \pi}{2^{j}}\right)$ for $p_{1}, p_{2} \in\left\{0,1, \ldots, 2^{j}-1\right\}$ (natural ordering).

2) Compute, from Eqs. (2), (7) and (8) the index $n \in$ $\left\{0,1, \ldots, 4^{j}-1\right\}$ (corresponding to the wavelet packet ordering) associated with $\left(p_{1}, p_{2}\right)$.

3) Set, for any pair $\left(p_{1}, p_{2}\right)$ given in step 1) and the corresponding $n$ obtained from step 2),

$$
\widehat{\gamma}\left(\frac{p_{1} \pi}{2^{j}}, \frac{p_{2} \pi}{2^{j}}\right)=\operatorname{var}\left[c_{j, n}^{r}\right] .
$$

From a practical point of view, the estimator of $\gamma\left(\frac{p_{1} \pi}{2^{j}}, \frac{p_{2} \pi}{2^{j}}\right)$ given by Eq. (29) assumes $i$ ) approximating the Shannon wavelet by a suitable wavelet and $i$ i) estimating the variance of $c_{j, n}$.

Approximating the Shannon wavelet can be performed by using Daubechies wavelets, depending to the Daubechies filter order $r$ (see Eq. (5)), the larger the order, the more relevant the approximation. In practice, Daubechies filters 
with $r=7,8$ yield relevant approximations of the Shannon filters.

Estimating the variance $\operatorname{Var}\left[c_{j, n}\right]$ of $c_{j, n}$ can be performed by using the standard unbiased variance estimator (also called unbiased sample variance). In the Section dedicated to the experimental results, $\operatorname{var}\left[c_{j, n}\right]$ will denote this unbiased estimator when formula of Eq. (29) is under consideration.

Assuming that an unbiased variance estimator is used, the bias of the above sample spectrum estimator depends on $j, r$ and the spectrum $\gamma$ shape. More precisely, we have:

Proposition 3: Let $X$ be a zero-mean random field having Shannon wavelet packet autocorrelation function of the form given by Eq. (25). Let $p_{1}, p_{2} \in\left\{0,1, \ldots, 2^{j}-1\right\}$. Let $n$ be the frequency index associated with $p_{1}, p_{2}$ in the sense of the correspondence $(j, n) \equiv\left(j,\left[n_{1}, n_{2}\right]\right)=$ $\left(j,\left[G^{-1}\left(p_{1}\right), G^{-1}\left(p_{2}\right)\right]\right)$ given in Section II.

Assume that $\gamma$ is continuous on $\Delta_{j, p_{1}}^{+} \times \Delta_{j, p_{2}}^{+}$and differentiable on the interior of $\Delta_{j, p_{1}}^{+} \times \Delta_{j, p_{2}}^{+}$. Assume that the standard unbiased estimator of variance is used in Eq. (29). We have:

$$
\left|\operatorname{Bias}\left[\widehat{\gamma}\left(\frac{p_{1} \pi}{2^{j}}, \frac{p_{2} \pi}{2^{j}}\right)\right]\right| \leqslant \pi C_{\nabla \gamma} \frac{\mathfrak{e}}{2^{j}}+\frac{C_{\gamma}}{4 \pi^{2}} \varepsilon_{j, n}^{r},
$$

where constants $\mathfrak{e}=(\sqrt{2}+\ln (1+\sqrt{2})) / 3<1, \quad C_{\nabla \gamma}=$ $\sup _{\Delta_{j, p_{1}}^{+} \times \Delta_{j, p_{2}}^{+}}\|\nabla \gamma\|, C_{\gamma}=\sup _{\Delta_{j, p_{1}}^{+} \times \Delta_{j, p_{2}}^{+}}|\gamma|$ and

$$
\varepsilon_{j, n}^{r} \leqslant\left.\int_{\mathbb{R}^{2}}|| \mathscr{F} W_{j, n}^{r}\left(\omega_{1}, \omega_{2}\right)\right|^{2}-\left|\mathscr{F} W_{j, n}^{\mathrm{S}}\left(\omega_{1}, \omega_{2}\right)\right|^{2} \mid \mathrm{d} \omega_{1} \mathrm{~d} \omega_{2} .
$$

The proof sketch is the following.

Proof: The bias of the estimator given by Eq. (29) is:

$$
\begin{array}{r}
\operatorname{Bias}\left[\widehat{\gamma}\left(\frac{p_{1} \pi}{2^{j}}, \frac{p_{2} \pi}{2^{j}}\right)\right]=\mathbb{E}\left[\operatorname{var}\left[c_{j, n}^{r}\right]\right]-\gamma\left(\frac{p_{1} \pi}{2^{j}}, \frac{p_{2} \pi}{2^{j}}\right) \\
=\operatorname{Bias}\left[\operatorname{var}\left[c_{j, n}^{r}\right]\right]+\operatorname{Var}\left[c_{j, n}^{r}\right]-\gamma\left(\frac{p_{1} \pi}{2^{j}}, \frac{p_{2} \pi}{2^{j}}\right) .
\end{array}
$$

Thus, if we consider the standard unbiased estimator of variance (Bias $\left[\operatorname{var}\left[c_{j, n}^{r}\right]\right]=0$ ), we obtain:

$$
\begin{aligned}
\left|\operatorname{Bias}\left[\widehat{\gamma}\left(\frac{p_{1} \pi}{2^{j}}, \frac{p_{2} \pi}{2^{j}}\right)\right]\right| \leqslant & \left|\operatorname{Var}\left[c_{j, n}^{r}\right]-\operatorname{Var}\left[c_{j, n}^{\mathrm{S}}\right]\right| \\
& +\left|\operatorname{Var}\left[c_{j, n}^{\mathrm{S}}\right]-\gamma\left(\frac{p_{1} \pi}{2^{j}}, \frac{p_{2} \pi}{2^{j}}\right)\right| .
\end{aligned}
$$

On one hand, we have

$$
\begin{aligned}
& \left|\operatorname{Var}\left[c_{j, n}^{r}\right]-\operatorname{Var}\left[c_{j, n}^{\mathrm{S}}\right]\right| \leqslant \frac{C_{\gamma}}{4 \pi^{2}} \times \\
& \left.\quad \int_{\mathbb{R}^{2}}|| \mathscr{F} W_{j, n}^{r}\left(\omega_{1}, \omega_{2}\right)\right|^{2}-\left|\mathscr{F} W_{j, n}^{\mathrm{S}}\left(\omega_{1}, \omega_{2}\right)\right|^{2} \mid \mathrm{d} \omega_{1} \mathrm{~d} \omega(33)
\end{aligned}
$$

On the other hand, we have

$$
\begin{aligned}
\mid \operatorname{Var}\left[c_{j, n}^{\mathrm{S}}\right] & -\gamma\left(\frac{p_{1} \pi}{2^{j}}, \frac{p_{2} \pi}{2^{j}}\right) \mid \\
& \left.=\frac{2^{2 j}}{\pi^{2}}\left|\int_{\Delta_{j, p_{1}}^{+} \times \Delta_{j, p_{2}^{+}}^{+}}\right| \gamma\left(\omega_{1}, \omega_{2}\right)-\gamma\left(\frac{p_{1} \pi}{2^{j}}, \frac{p_{2} \pi}{2^{j}}\right)\right] \mid \mathrm{d} \omega_{1} \mathrm{~d} \omega_{2} \\
& \leqslant \frac{2^{2 j}}{\pi^{2}} \int_{\Delta_{j, p_{1}}^{+} \times \Delta_{j, p_{2}}^{+}} \gamma\left(\omega_{1}, \omega_{2}\right)-\gamma\left(\frac{p_{1} \pi}{2^{j}}, \frac{p_{2} \pi}{2^{j}}\right) \mid \mathrm{d} \omega_{1} \mathrm{~d} \omega(234)
\end{aligned}
$$

Since $\gamma$ is continuous on $\Delta_{j, p_{1}}^{+} \times \Delta_{j, p_{2}}^{+}$and differentiable on the interior of $\Delta_{j, p_{1}}^{+} \times \Delta_{j, p_{2}}^{+}$, we have, from the mean value theorem:

$$
\begin{aligned}
& \left|\gamma\left(\omega_{1}, \omega_{2}\right)-\gamma\left(\frac{p_{1} \pi}{2^{j}}, \frac{p_{2} \pi}{2^{j}}\right)\right| \leqslant \\
& \sup _{\left(u_{1}, u_{2}\right) \in \Delta_{j, p_{1}}^{+} \times \Delta_{j, p_{2}}^{+}}\left\|\nabla \gamma\left(u_{1}, u_{2}\right)\right\| \times \sqrt{\left(\omega_{1}-\frac{p_{1} \pi}{2^{j}}\right)^{2}+\left(\omega_{2}-\frac{p_{2} \pi}{2^{j}}\right)^{2}}
\end{aligned}
$$

for any $\left(\omega_{1}, \omega_{2}\right) \in \Delta_{j, p_{1}}^{+} \times \Delta_{j, p_{2}}^{+}$, with

$$
\int_{\Delta_{j, p_{1}}^{+} \times \Delta_{j, p_{2}}^{+}} \sqrt{\left(\omega_{1}-\frac{p_{1} \pi}{2^{j}}\right)^{2}+\left(\omega_{2}-\frac{p_{2} \pi}{2^{j}}\right)^{2}} \mathrm{~d} \omega_{1} \mathrm{~d} \omega_{2}=\mathfrak{e}\left(\frac{\pi}{2^{j}}\right)^{3} .
$$

Proposition 3 then follows from Eqs. (32), (33), (34), (35) and (36).

From Proposition 3, we derive that the bias of the estimator given by Eq. (29) depends on the decomposition level and wavelet order used. This bias tends to 0 when both $j$ and $r$ tends to infinity. Notice that $\varepsilon_{j, n}^{r}$ tends to 0 as $r$ tends to infinity, $\forall j, n$, due to the convergence of the filter $\mathbf{H}_{j, n}^{r}$ to the Shannon filter $\mathbf{H}_{j, n}^{\mathrm{S}}$ as $r$ tends to infinity.

\section{EXPERIMENTAL RESUlTS}

This section provides experimental results on spectral analysis of textures. Section V-A addresses the quality of the wavelet packet spectrum estimator to capture textural information. Section V-B provides quantitative evaluation of the wavelet packet spectrum estimator in the framework of content based image retrieval.

\section{A. Spectral analysis and spectral texture contents}

Wavelet packet spectra ${ }^{1}$ of some texture images are provided in Figures 1 and 2. The wavelet packet spectra have been computed from the method given in Section IV-B, where the decomposition level is 6 and the Daubechies wavelet with order $r=7$ is used. Spectra computed from the Fourier transform are also given in this figure, for comparison purpose.

From a visual analysis of images given in Figures 1 and 2 (by focusing on image interpretation without watching spectra given in the same figures), one can remark that most of these textures exhibit non-overlapping textons replicating repeatedly: thus, coarsely, we can distinguish several frequencies having significant variance contributions (from a theoretical consideration), when the texture does not reduce to the replications of a single texton.

In addition, when these textons occupy approximately the same spatial area (see for instance "Fabric" textures in Figure 2), the frequencies with high variance contributions (peak in the spectrum) are close in terms of their spatial location (from a theoretical consideration).

\footnotetext{
${ }^{1}$ Colors represented in Figures 1 and 2 are simulated from a light source in order to ease 3-D visualization: red color [value 1] corresponds to fully illuminated shapes whereas blue color [value 0] is associated to shaded areas, green color corresponds to value 0.5 .
} 

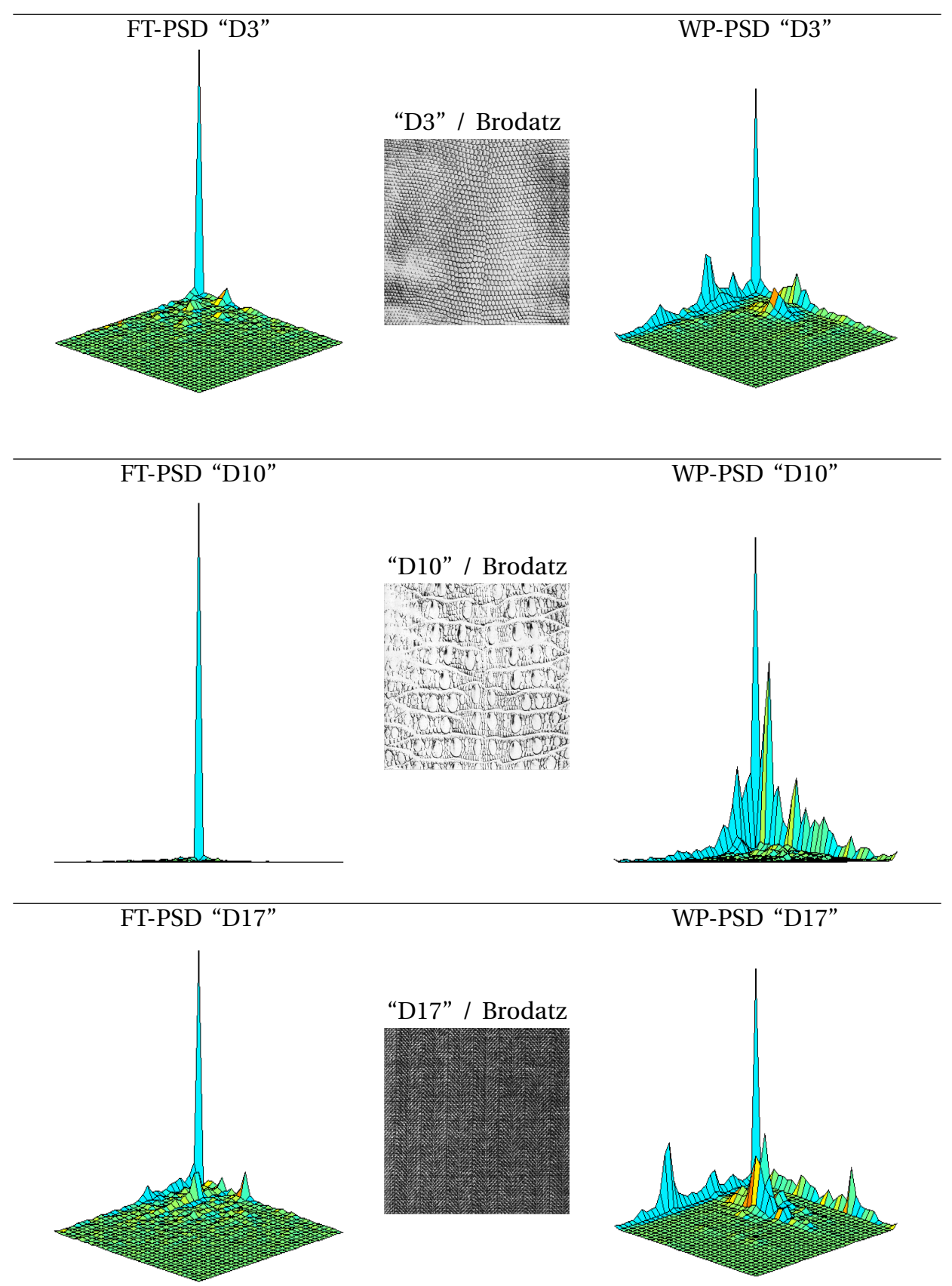

Fig. 1. Textures images and their spectra $\widehat{\gamma}$ computed by using discrete Fourier and wavelet packet transforms. Abscissa of the spectra images consist of a regular grid over $[0, \pi / 2] \times[0, \pi / 2]$.

The above heuristics, issued from visual image analysis, are confirmed by considering the wavelet packet spectra (see for instance spectra of "Fabric" textures in Figure 2), whereas, in most cases, the two dimensional discrete Fourier transform exhibits only one peak.

One can highlight that the poorness of the Fourier spectra is not due to a lack of resolution in the sampling step of the Fourier transform. This poorness can be explained by noting that Fourier transform is sensitive to global spatial regularity. In contrast wavelet packets can capture local spatial regularity and lead to a more informative spectrum estimator when several frequencies contribute in texture variance distribution.

Remark 2: In the above experimental results, we have considered a regular frequency grid over $[0, \pi] \times[0, \pi]$. Consequently, we have performed a uniform sampling of the spectrum of the input texture.

Note that when we have some $a$ priori on the spectrum shape, we can apply a non-uniform sampling scheme by simply focusing on the particular tree structure associated with the subbands-of-interest. These subbands are those associated with wavelet packet functions having tight Fourier transform support across the frequencies where the spectrum exhibits sharp components. 

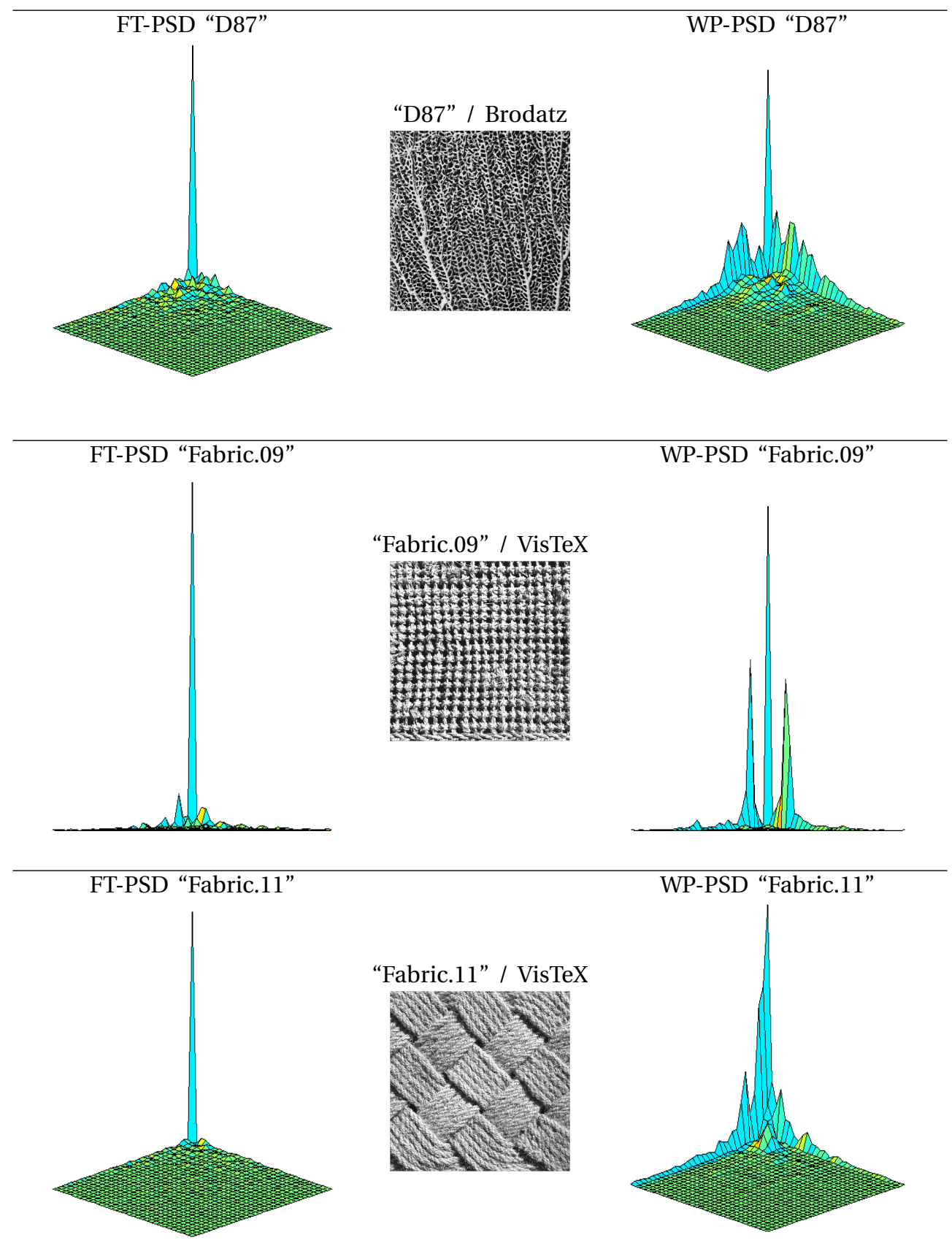

Fig. 2. Textures images and their spectra $\widehat{\gamma}$ computed by using discrete Fourier and wavelet packet transforms. Abscissa of the spectra images consist of a regular grid over $[0, \pi / 2] \times[0, \pi / 2]$.

As a matter of example: by considering the spectrum of texture "D87" (see Figure 2), we can estimate accurately this spectrum by using a large amount of samples in $[0, \pi / 4] \times[0, \pi / 4]$ and very few samples in $[0, \pi] \times[0, \pi] \backslash$ $[0, \pi / 4] \times[0, \pi / 4]$. The corresponding wavelet packet decomposition concerns fewer subbands than a full wavelet packet decomposition and is thus with less computational complexity.
$B$. Content based image retrieval by using spectral texture features

Section V-A have shown qualitatively that wavelet packets are relevant in analyzing textures with rich spectral content. In this section, we evaluate the contribution of wavelet packets in Content Based Image Retrieval (CBIR) from a database composed with such textures.

The database under consideration is composed with $M=94$ images associated with the 6 texture classes given in Figures 1 and 2. These $M$ images are obtained from the splitting of every 'large' texture image into 16 nonoverlapping images. An image from the database thus has 
$160 \times 160$ pixels when it pertains to one of the classes "D3", "D10", "D17" or "D87" and $128 \times 128$ pixels for classes "Fabric.09" and "Fabric.11" (the larger images have $640 \times 640$ pixels in the Brodatz album and $512 \times 512$ pixels in the VisTeX album).

In order to compare a query image $I_{q}$ with an arbitrary image $I_{\ell}, 1 \leqslant q, \ell \leqslant M$, we use the Log-Spectral distance and the symmetric version of the Kullback-Leibler divergence. For images $I_{1}, I_{2}$ with sample spectra $\gamma_{1}, \gamma_{2}$, their LogSpectral distance is given by

$$
L S\left(\gamma_{1}, \gamma_{2}\right) \propto\left(\sum_{q, \ell} \log \left(\frac{\gamma_{1}^{2}\left(\omega_{q}, \omega_{\ell}\right)}{\gamma_{2}^{2}\left(\omega_{q}, \omega_{\ell}\right)}\right)\right)^{1 / 2}
$$

and their symmetric Kullback-Leibler divergence is defined by

$$
K L\left(\gamma_{1}, \gamma_{2}\right)=K L\left(\gamma_{1} \| \gamma_{2}\right)+K L\left(\gamma_{2} \| \gamma_{1}\right)
$$

where

$$
K L\left(\gamma_{1} \| \gamma_{2}\right) \propto \sum_{q, \ell} \gamma_{1}\left(\omega_{q}, \omega_{\ell}\right) \log \left(\frac{\gamma_{1}\left(\omega_{q}, \omega_{\ell}\right)}{\gamma_{2}\left(\omega_{q}, \omega_{\ell}\right)}\right)
$$

Table I provides the CBIR retrieval rates per texture and the average CBIR retrieval rate over the 6 texture classes. In this table, the above spectral similarity measurements are computed upon discrete Fourier, wavelet and wavelet packet spectra. The Daubechies wavelet of order $r=7$ and $j=4$ have been used to compute wavelet and wavelet packet spectra from Eq. (29).

This table emphasizes

- the suitability of wavelet based approaches, in comparison with the Fourier approach,

- the sensitivity of the wavelet transform to the similarity measures considered, which may follow from the coarse-irregular spectrum sampling induced by using wavelet method.

- the good performance of the wavelet packet spectral analysis for the Log-Spectral and Kullback-Leibler similarity measurements.

One can note that the wavelet transform is a particular case of the wavelet packet transform so that the wavelet spectrum can be seen through the wavelet packet spectrum by zooming on the neighborhood of the zero frequency. This wavelet spectrum thus follows from a non-uniform sub-sampling of the wavelet packet spectrum (non-regular frequency grid). From similarity evaluations, this sampling scheme can be interpreted as a binary sparse weighting, where the non-zero values are concentrated in low frequencies. This sampling scheme consequently penalizes highly medium and high frequencies. This strategy is suitable for textures with spectral supports localized in the neighborhood of zero (up to $90 \%$ of textures from natural images). However, this strategy can justify the contrasted results obtained by using the wavelet transform for the tests performed upon the database of Table I: the spectral content of these textures is not restricted to low frequencies.

In addition to the above binary weighting regarding spectra sample points, the log function involved in LogSpectral and Kullback-Leibler measures impacts differently the sample spectra $\gamma_{1}\left(\omega_{q}, \omega_{\ell}\right), \gamma_{2}\left(\omega_{q}, \omega_{\ell}\right)$ under comparison. Indeed, for large values of $\gamma_{1}\left(\omega_{q}, \omega_{\ell}\right) / \gamma_{2}\left(\omega_{q}, \omega_{\ell}\right)$, the log function imposes a higher penalty in Kullback-Leibler measurements than in Log-Spectral measurements.

TABLE I

RETRIEVAL RESULTS PER TEXTURE AND AVERAGE RETRIEVAL RESULTS FOR THE 6 TEXTURE CLASSES GIVEN IN FiguRES 1 AND 2. EXPERIMENTAL RESUltS ARE PERFORMED WITH THE LOG-SPECTRAL SPECTRAL DISTANCE AND THE Kullback-Leibler Divergence. Specifically, FT, WT and WPT

\begin{tabular}{|c|c|c|c|c|c|c|}
\hline Measure: & \multicolumn{3}{|c|}{ Log-Spectral } & \multicolumn{3}{|c|}{ Kullback-Leibler } \\
\hline \begin{tabular}{|l|} 
Texture \\
\end{tabular} & FT & WT & WPT & FT & WT & WPT \\
\hline D3 & 44.5 & 88.7 & 99.2 & 39.5 & 58.2 & 91.0 \\
\hline D10 & 71.5 & 82.8 & 90.2 & 90.6 & $\mathbf{5 8 . 2}$ & 85.6 \\
\hline D17 & 93.0 & 100 & 99.6 & 100 & 99.6 & 100 \\
\hline D87 & 77.3 & 100 & 96.1 & 87.9 & 87.5 & 99.2 \\
\hline Fabr.09 & 84.4 & 100 & 83.6 & 82.8 & 80.5 & 98.1 \\
\hline Fabr.11 & 69.5 & 95.7 & 77.0 & 74.6 & 73.4 & 97.3 \\
\hline
\end{tabular}
Designate the discrete Fourier, Wavelet and Wavelet PaCket TRANSFORMS, RESPECTIVELY.

\section{Conclusion}

Two issues are addresses in the paper: $(i)$ associating a PSD to the wavelet packet coefficients of random fields and (i i ) estimating this PSD from the statistical properties of the wavelet packet coefficients.

Issue $(i)$ has been addressed for stationary random fields and some non-stationary fractional Brownian fields. Issue ( $i$ i ) has been tackled from asymptotic properties of the Shannon wavelet packets and the spectrum estimation method proposed is more effective for wavelet filters with large order.

The PSD estimation method derived in the paper has shown relevancy in estimating the PSD of texture images. This method outperforms Fourier based spectral estimation when the textures under consideration have substantial spectral content (when spectrum do not reduces to a peak at single frequency).

Prospects regarding this work may concern:

$\left(p_{1}\right)$ The extension of the method to adaptive sampling of the PSD: a best basis can be derived in order to achieve an efficient strategy. Indeed, the experimental results have shown that for many textures, a large frequency domain is unoccupied whereas estimating several spectrum sample points in this domain could increase estimation errors due to the decimation step involved by the wavelet packet decomposition.

$\left(p_{2}\right)$ The extension of the method with respect to nonorthogonal wavelet packet transforms: the redundancy of these transforms will probably benefit to the spectrum estimation. Note that the spectrum needs to be defined with respect to the transform and its estimation cannot reduce to variances per subbands unless any given subband has been processed accordingly.

Some applications of the method are:

$\left(a_{1}\right)$ Hurst parameter estimation for self-similar medical images, see for instance [2]. 
$\left(a_{2}\right)$ texture modeling by using Wold decompositions [17], [18]: estimation the poles of the spectrum is necessary to determine the spectral singularities involved in the deterministic texture contribution. These poles are associated with peaks of the spectrum and their number, as well as their location determines the accuracy of the modeling.

$\left(a_{3}\right)$ Spread-spectrum image watermarking [19], [20], [21], [22], [23], textural change detection by using spectral distances [24], among other references.

We conclude by noting that the wavelet packet decomposition tree can be chosen adaptively with respect to the spectrum shape in order to makes possible non-uniform sampling schemes: the analysis performed in the paper suggests estimating the spectrum from 1) a full wavelet packet decomposition especially for applications involving images with rich spectral contents (for instance when the input image has many spectral singularity points, as in the case of the multifractional brownian fields) and 2) a sub-tree of the full wavelet packet tree, this sub-tree being deleafed in frequency domains associated with less spectral information (in particular, a wavelet spectrum follows from a non-uniform sampling grid associated with a tree that expands mainly in low frequencies).

\section{ACKNOWLEDGEMENT}

The authors address special thanks to the anonymous reviewers and the associate editor for their constructive comments.

\section{REFERENCES}

[1] D. Mondal and D. B. Percival, "Wavelet variance analysis for random fields on a regular lattice," IEEE Transactions on Image Processing, vol. 21, no. 2, pp. 537 - 549, Febr. 2012.

[2] O. Nicolis, P. Ramirez-Cobo, and B. Vidakovic, "2d wavelet-based spectra with applications," Computational Statistics and Data Analysis, vol. 55, no. 1, pp. $738-751,2011$.

[3] I. A. Eckley, G. P. Nason, and R. L. Treloar, "Locally stationary wavelet fields with application to the modelling and analysis of image texture," Journal of the Royal Statistical Society: Series C (Applied Statistics), vol. 59, no. 4, pp. 595-616, 2010. [Online]. Available: http://dx.doi.org/10.1111/j.1467-9876.2009.00721.x

[4] P. Porwik and A. Lisowska, "The haar-wavelet transform in digital image processing: Its status and achievements," Machine graphics and vision, vol. 13, no. 1-2, pp. 79 - 98, 2004.

[5] A. M. Atto, D. Pastor, and G. Mercier, "Wavelet packets of fractional brownian motion: Asymptotic analysis and spectrum estimation," IEEE Transactions on Information Theory, vol. 56, no. 9, pp. 4741 - 4753, Sep. 2010.
[6] M. V. Wickerhauser, Adapted Wavelet Analysis from Theory to Software. AK Peters, 1994.

[7] S. Mallat, A wavelet tour of signal processing, second edition. Academic Press, 1999.

[8] I. Daubechies, Ten lectures on wavelets. SIAM, Philadelphie, PA, 1992.

[9] P. F. Craigmile and D. B. Percival, "Asymptotic decorrelation of between-scale wavelet coefficients," IEEE Transactions on Information Theory, vol. 51, no. 3, pp. 1039 - 1048, Mar. 2005.

[10] T. Kato and E. Masry, "On the spectral density of the wavelet transform of fractional brownian motion," Journal of Time Series Analysis, vol. 20, no. 50, pp. 559 - 563, 1999.

[11] R. Averkamp and C. Houdré, "Some distributional properties of the continuous wavelet transform of random processes," IEEE Transactions on Information Theory, vol. 44, no. 3, pp. 1111 - 1124, may 1998.

[12] S. Cambanis and C. Houdré, "On the continuous wavelet transform of second-order random processes," IEEE Transactions on Information Theory, vol. 41, no. 3, pp. 628 - 642, may 1995.

[13] P. Flandrin, "Wavelet analysis and synthesis of fractional brownian motion," IEEE Transactions on Information Theory, vol. 38, no. 2, pp. 910 - 917, Mar. 1992.

[14] A. M. Atto and Y. Berthoumieu, "Wavelet packets of nonstationary random processes: Contributing factors for stationarity and decorrelation," IEEE Transactions on Information Theory, vol. 58, no. 1, pp. 317 - 330, Jan. 2012.

[15] C. S. Burrus, R. A. Gopinath, and H. Guo, Introduction to Wavelets and Wavelet Transforms: A Primer. Prentice Hall, 1998.

[16] Y. Meyer, Wavelets, Algorithms and Applications. SIAM, Philadelphie, 1993.

[17] F. Liu and R. W. Picard, "Periodicity, directionality, and randomness: Wold features for image modeling and retrieval," IEEE Transactions on Pattern Analysis and Machine Intelligence, vol. 18, pp. 722 - 733, 1996.

[18] Y. Stitou, F. Turcu, Y. Berthoumieu, and M. Najim, "Three-dimensional textured image blocks model based on wold decomposition," IEEE Transactions on Signal Processing, vol. 55, no. 7, pp. 3247 - 3261, 2007.

[19] A. Bors and I. Pitas, "Image watermarking using block site selection and DCT domain constraints," Optic Express, vol. 3, no. 12, pp. 512 - 523, Dec 1998. [Online]. Available: http://www.opticsexpress.org/abstract.cfm?URI=oe-3-12-512

[20] S.-H. Wang and Y.-P. Lin, "Wavelet tree quantization for copyright protection watermarking," IEEE Transactions on Image Processing, vol. 13, no. 2, pp. 154 - 165, Febr. 2004.

[21] M. Gkizeli, D. A. Pados, and M. J. Medley, "Optimal signature design for spread-spectrum steganography," IEEE Transactions on Image Processing, vol. 16, no. 2, pp. $391-405$, Febr. 2007.

[22] R. Kwitt, P. Meerwald, and A. Uhl, "Lightweight detection of additive watermarking in the DWT-domain," IEEE Transactions on Image Processing, vol. 20, no. 2, pp. 474 - 484, Febr. 2011.

[23] L. Cui and W. Li, "Adaptive multiwavelet-based watermarking through jpw masking," IEEE Transactions on Image Processing, vol. 20, no. 4, pp. 1047 - 1060, April 2011

[24] C. T. Vu, T. D. Phan, and D. M. Chandler, "S3: a spectral and spatial measure of local perceived sharpness in natural images," IEEE Transactions on Image Processing, vol. 21, no. 3, pp. 934 - 945, March 2012. 